\title{
Frontières
}

\section{La polyvalence du moi tragique}

La portée idéologique des dernières volontés chez les tragiques grecs

\section{Essam Safty}

Volume 21, numéro 1, automne 2008

Prévenir le suicide

URI : https://id.erudit.org/iderudit/037885ar

DOI : https://doi.org/10.7202/037885ar

Aller au sommaire du numéro

\section{Éditeur(s)}

Université du Québec à Montréal

ISSN

1180-3479 (imprimé)

1916-0976 (numérique)

Découvrir la revue

Citer cet article

Safty, E. (2008). La polyvalence du moi tragique : la portée idéologique des dernières volontés chez les tragiques grecs. Frontières, 21(1), 134-139. https://doi.org/10.7202/037885ar

\section{Résumé de l'article}

Les dernières volontés furent l'occasion, pour les héros de la tragédie grecque, de dire la diversité du moi tragique à travers ses agitations in articulo mortis. Moyennant l'accomplissement d'un geste sacramentel qui tient lieu d'une promesse se dressant contre les incertitudes du devenir, elles permirent en effet aux héros tragiques d'exalter le moi physique, de prévenir l'attentat à l'intégrité de ce dernier, d'en prolonger les affections, soit directement ou par procuration, comme dans le cas des voeux de vengeance, où le mourant devient a posteriori sujet agissant dans le moi d'un autre sujet, soit au moyen d'un acte dicté par les impératifs mêmes de l'éthique de telle ou telle passion, telle que l'amour, qui, suivant sa définition mytho-idéologique, fait du sujet aimant à la fois un moi-même et un autre. 


\section{Résumé}

Les dernières volontés furent l'occasion, pour les héros de la tragédie grecque, de dire la diversité du moi tragique à travers ses agitations in articulo mortis. Moyennant l'accomplissement d'un geste sacramentel qui tient lieu d'une promesse se dressant contre les incertitudes du devenir, elles permirent en effet aux héros tragiques d'exalter le moi physique, de prévenir l'attentat à l'intégrité de ce dernier, d'en prolonger les affections, soit directement ou par procuration, comme dans le cas des vœux de vengeance, où le mourant devient a posteriori sujet agissant dans le moi d'un autre sujet, soit au moyen d'un acte dicté par les impératifs mêmes de l'éthique de telle ou telle passion, telle que l'amour, qui, suivant sa définition mytho-idéologique, fait du sujet aimant à la fois un moi-même et un autre.

Mots clés: dernières volontés - mort sépulture - tragédie grecque le moi tragique - poignée de main vengeance - amour - eschatologie.

\begin{abstract}
Last wills have provided Greek Tragedy heroes with the opportunity to express the diversity of the agitated tragic self in articulo mortis. Provided the accomplishment of a sacramental gesture which serves as a defying promise against future uncertainties, they allowed the tragic heroes to exalt the physical self, to prevent dishonoring the plenitude of the latter, as well as to prolong its affections, either directly or indirectly, as in the case of revenge vows, wherein the dying hero becomes a posteriori an acting agent in another subject's self. The dying hero's affections may also be prolonged through an act which is prompted by the very ethical demands of a given passion, such as love, which, according to its mythoideological definition, makes the loving subject both a self and another.
\end{abstract}

Keywords: Last wills - death sepulchre - Greek Tragedy - the tragic Self - Handshake - Revenge - Love eschatology.

\section{La polyvalence du moi tragique La portée idéologique des dernières volontés chez les tragiques grecs}

\author{
Essam Safty, D. ès L., \\ professeur titulaire, St. Thomas University, \\ Nouveau-Brunswick.
}

Sans doute les dernières volontés sontelles l'occasion d'une exaltation des plus enthousiastes du «moi » : le «je » n'est pas un autre, mais plutôt affirmation identitaire et participation active à la prolongation du moi. Moyen détourné de joindre l'en deçà à l'au-delà, les dernières volontés constituent en effet le testament verbal grâce auquel le mourant continuera d'être, une fois qu'il ne sera plus, sujet agissant et ce, en se signalant précisément par son absence. Ce jeu fin est en somme une certaine assurance que l'on prend en vue de sa propre survie dans la conscience d'autrui.

Or, si la diversité du moi tragique appelle plus d'une nuance, la question des dernières volontés engage d'emblée celle de leur exécution; donc l'assurance prise par le mourant prend de prime abord la forme d'une promesse qui émane d'un autre sujet éthique «autonome et supra moral» tel que l'individu nietzschéen, qui voit dans l'accomplissement de la promesse non pas le sacrifice de soi, mais la manifestation de la force qui domine en soi. Cette vision du sujet qui promet est bien conforme à l'éthique du héros tragique, qui demeure par définition seul maître de son devenir. On la retrouve, depuis l'Iliade d'Homère (VI, v. 233), les Nuées d'Aristophane (v. 81), et, dès le $\mathrm{IV}^{\mathrm{e}}$ siècle avant J.-C., dans les stèles funéraires (voir Hans Diepolder, 1931), sous une forme symbolique et sacramentelle: la poignée de main ${ }^{1}$. Hercule par exemple, demandant à Hyllos, son fils, de transporter son corps sur l'Eta, lui dit: «Donne-moi $[\ldots]$ ta main droite $[\ldots]$; jure sur la tête de Zeus [...] d'exécuter point par point mes instructions» (Sophocle, Trachiniennes, v. 1177 s.); le Philoctète du même poète, ayant formulé le vœu d'être plongé dans les fournaises de Lemnon, dit à Néoptolème, qui avait accepté d'accéder au vœu de son père: "Ta main, en gage de ta promesse » (Philoctète, v. 815-816); l'ÆEdipe du même poète également, prêt à mourir, ou plutôt à être élevé au rang des consacrés, adresse à Thésée la pieuse requête que voici: "Cher ami, donne la main à mes enfants, en gage de l'antique lien [...] Promets que jamais tu ne les abandonneras» (Edipe à Colone, v. 1636-1637). Oreste, dans l'Iphigénie en Tauride d'Euripide, dit à Pylade, son compagnon d'infortune: «[...] accomplis la mission qu'en pressant ta main droite je confie à ta foi ; élève-moi un tombeau [...] (v. 699704). Ailleurs, à Hélène, qui lui promet de mourir «plutôt que de prendre un autre époux», le Ménélas du même poète s'écrie : «Ah! Pour sceller cette promesse, mets ta main droite dans la mienne» (Hélène, v. 838). 
Cela dit, la question de la garantie éthique de l'accomplissement des dernières volontés résolue, il convient à présent de relever que le jeu fin signalé plus haut se traduit préalablement par la volonté de repousser les limites temporelles du moi. La mort en sursis est en effet l'aboutissement de tant d'efforts consentis par quantité de héros tragiques en vue de jouir quelques instants de plus de ces biens universels que sont la lumière du soleil et l'harmonie de la voûte céleste: la déchirure du moi physique n'était que partie remise, et celui-ci devra se résoudre, comme on le verra, à se survivre autrement. Mourant, l'homme ancien regrettait effectivement la beauté de la voûte éthérée, le charme de la nature et les bienfaits de la lumière. L'attachement du moi au monde sensible, c.-à-d. l'amour de la vie, trouvait ainsi ses accents les plus pathétiques, son expression la plus enthousiaste et ses élans les plus tendres lorsqu'il se trouvait confondu avec l'idée de la privation de la lumière céleste; car, après tout, «nous sommes tous, affirme Plutarque, qui invoque l'autorité d'Euripide, impatiemment amoureux et désireux de voir "ce beau soleil qui éclaire la terre" »(Les ouvres morales et mêlées de Plutarque, t. I, v. 290 A). Ainsi : "Ô lumière, gémit Ajax, alors qu'il s'apprête à s'abattre ${ }^{2}$ sur la pointe de son épée, ô sol sacré de Salamine où je suis né [...] et vous sources, rivières [...], adieu, tout ce qui a été ma vie! Vous avez entendu les ultimes paroles d'Ajax: je n'ouvrirai plus la bouche que devant les ombres» (Sophocle, Ajax, v. 860 s.). Ailleurs, c'est dans la bouche de son Iphigénie qu'Euripide place ces cris d'alarme: "C'en est fini pour moi de la lumière et de ce soleil éclatant " (Iphigénie à Aulis, v. 1281-1282); à la servante qui nous apprend la nouvelle de la mort de son Alceste, le même Euripide fait dire: «Défaillante [...], d'un souffle [...] bien faible, vers la clarté du jour elle veut tourner les yeux. Car plus jamais elle ne la verra; pour la dernière fois aujourd'hui, elle va contempler les rayons et l'orbe du soleil» (Alceste, v. 204-209); à son Héraclès, qui, revenant des Enfers, est frappé de voir les siens sombrés dans un profond chagrin, le même poète encore fait dire: "Arrachez de votre tête ces bandelettes funèbres, et levez les yeux vers la lumière: après les ténèbres infernales, le changement est doux aux yeux qui la contemplent» (Héraclès furieux, v. 562-563) 3 . Le rapport est confirmé par Plutarque: "Ceux qui ont longuement demeuré en ténèbres ne peuvent soudainement supporter ni endurer la lumière des rayons du Soleil » (Les œuvres morales et mêlées de Plutarque, t. I, v. 24 B). Ainsi, mourants ou renaissants à la lumière, c'est à Phébus que les Anciens ${ }^{4}$ adressaient leurs témoignages d'amour et de déférence. Jamais opposition entre le moi qui se meurt et le moi qui n'est plus, c.-à-d. entre vie et mort, ne pouvait mieux s'illustrer que par la dichotomie lumière / ténèbres. On s'explique donc la maxime de La Rochefoucauld, selon laquelle «Le soleil ni la mort ne se peuvent regarder fixement» (Maximes, maxime 26.47) ${ }^{5}$; et on comprend dès lors la raison pour laquelle Hercule demande pardon à Phébus d'avoir emporté des Enfers, et partant fait paraître à la face des cieux, un butin que le dieu ne devait point contempler ${ }^{6}$. C'est que, au fond, il existe une impossibilité idéologique qui découle de très vieilles conceptions selon lesquelles le Soleil passait pour être la divinité de la purification, et la Mort, une source de souillure.

Cela étant, ces adieux à la lumière opérant une réconciliation progressive du moi avec la déchirure apportée par la mort imminente, la préservation de l'intégrité du moi physique devient certes l'une des principales préoccupations des mourants; d'où, souvent, un obsédant soin contracté envers le sort de son propre cadavre. Le corps humain se trouvait être en effet, dans la conception guerrière des Anciens, pour qui robustesse et carrure étaient synonymes d'héroïsme, l'objet d'un véritable culte d'admiration: animé, il était symbole d'héroïsme actif et de grandeur royale ou guerrière; mais, inerte ou dépossédé de ses valeureux attributs, de sujet actif qu'il était, il devenait objet et spectacle: objet du regard d'autrui et des soins assurés par l'autre, mais aussi spectacle digne d'admiration. En somme, mort ou vivant, le corps humain ne cessait de fournir, dans les auteurs anciens, un beau sujet d'admiration: "[dans le corps d'un jeune homme], s'écrie Priam devant le vainqueur, tout est beau, même en son cadavre» (Homère, Iliade, XXII, v. 73). Le même Homère dit l'empressement de la foule autour du cadavre d'Hector: "Les fils d'Achéens accoururent [...] contemplant la taille et la beauté admirable d'Hector» (Homère, Iliade, XXII, v. 370-71). Et Euripide fait dire au chœur des mères des Sept Chefs, lesquelles venaient réclamer les corps de leurs enfants abandonnés sans sépulture, «Pour moi, revoir les membres de mes enfants est un spectacle cruel, beau cependant» (Suppliantes, v. 783).

Les héros anciens savaient donc qu'une fois morts, ils allaient devenir l'objet des curieux regards d'un autre; d'où précisément l'élaboration in extremis de toute une esthétique de la belle mort: mourant, le je tient à s'illustrer de quelque agrément, de quelque beauté, grâce ou marque d'honneur afin de graver dans la mémoire de l'autre le sceau d'une mort gracieuse, honorable et noble. À ce soin, qui dit la vanité du moi mourant et, de fait, allant au-devant de ses propres dernières volontés, peu de héros surent échapper. Sans doute la belle mort est-elle un fort honorable titre à la gloire, du moins posthume, pour le moi qui n'est plus: elle le déifie en quelque sorte et l'idéalise en le fixant dans la mémoire de l'autre. Ainsi, nous voyons Alceste, le matin où elle savait qu'elle devait mourir, s'administrer des soins destinés à souligner sa beauté dans la mort: "Elle fit sur son corps blanc ruisseler les eaux vives, rapporte une servante; puis des armoires de cèdre tirant vêtements et joyaux, elle se fit très belle» (Euripide, Alceste, v. 157 s.) $)^{7}$. Polyxène, quant à elle, tient à conserver sa pudeur virginale lors de l'ultime chute: «Ô vous, Argiens [...], que personne ne porte la main sur mon corps; je tendrai ma gorge avec courage.» Et Talthybios, qui vient d'assister au sacrifice de la vierge troyenne, de commenter : «Elle, dans la mort, conservait le souci de tomber avec décence, en dérobant aux regards ce que les hommes ne devaient pas voir» (Euripide, Hécube, v. 547 s. $)^{8}$. La généreuse Macarie embrasse, elle aussi, le même idéal de pudeur: "Que ce ne soit pas, insiste-t-elle, entre les mains des hommes mais entre celles des femmes que j'exhale ma vie » (Euripide, Héraclides, v. 564-565).

La belle mort participe également, par la façon même dont elle est accueillie ou saluée, de l'activisme héroïque du mourant, lequel, loin de s'effrayer, s'affaire à sa rencontre. La doctrine stoïcienne proscrit en effet toute forme d'oisiveté, et ce, jusqu'aux derniers instants de la vie d'un homme. C'est ce que rappelle Sénèque à Serenus dans son De l'oisiveté: "Chez nous, il est si vrai qu'il n'y a pas d'oisiveté avant la mort que, si les circonstances le permettent, notre mort même n'est pas oisive» (I, 4). Cela explique en effet la raison pour laquelle on voit Hercule, chez Sophocle, demander à son fils, Hyllos, de le tuer pour qu'il mette terme à ses souffrances (Trachiniennes, v. 1031 s.), alors que, chez Sénèque, on le voit aller, de plein gré, au-devant de la mort: gagné par le stoïcisme latin, au lieu d'exhorter son fils à embraser le bûcher sur lequel il devait être incinéré, le héros grec le fait lui-même. Et, devenu stoïcien ${ }^{9}$, il se garde, quoique mourant, d'un trépas oisif, en embrasant, de ses propres mains, les troncs qui restaient ininflammables dans son immense bûcher ${ }^{10}$. Cette grandeur d'âme et cette stupéfiante sérénité, dont témoigna l'Hercule latinisé au milieu des flammes de son bûcher, font précisément la beauté de son ultime épreuve et parachèvent en quelque sorte le glorieux cycle de ses Douze travaux. 


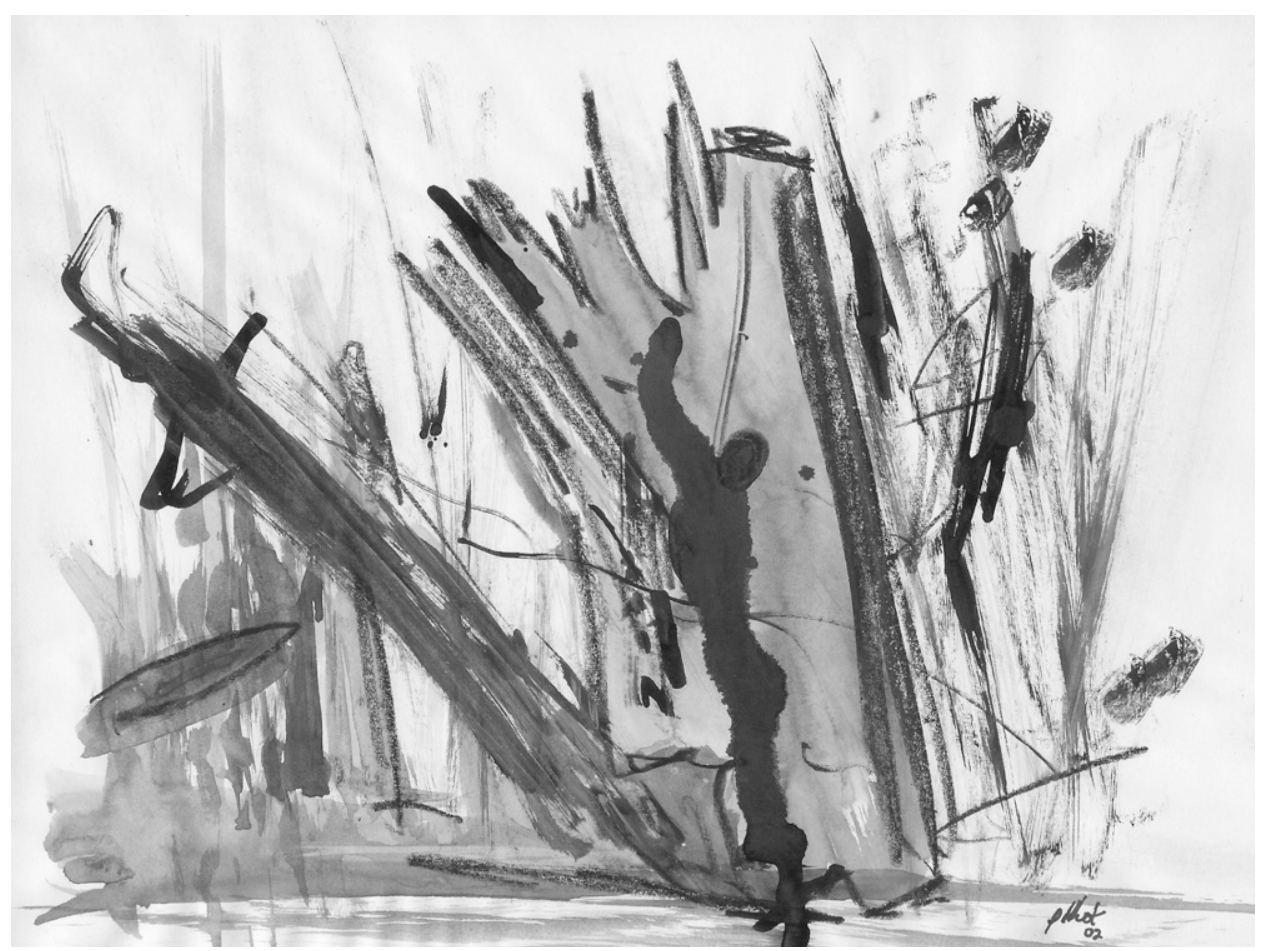

Cela dit, pour certains, ni beauté physique, ni pudeur, honneur, ou gloire ne font autorité dans la définition des critères de la belle mort: celle-ci peut être tout simplement une mort paisible et sereine, qui, ne brusquant pas ses victimes, les exempte des affres de l'agonie ${ }^{11}$. La Cassandre d'Eschyle par exemple, se sachant au bord de la mort, ne désire plus qu'une chose: «[...] un coup bien placé, dit-elle, pour fermer mes yeux sans convulsions en répandant doucement mon sang» (Agamemnon, v. 1293-1294). Sophocle fera formuler ce même vœu à son Ajax s'apprêtant à se donner la mort: « [...] j'invoque Hermès infernal, le guide des morts. Qu'il m'endorme doucement et que ce soit d'un saut facile et prompt que j'aille déchirer mon flanc à cette épée» (Ajax, v. 831-834). Et l'Héraclès du même poète, craignant d'être saisi de nouveau, lors de ses souffrances, de ses accès de convulsions, enjoint Hyllos de le porter au plus vite sur le bûcher où il doit être incinéré: "N'attends pas que m'assaillent de nouveaux spasmes et de nouvelles fureurs pour me placer sur le bûcher» (Trachiniennes, v. 1253 s.).

Ce culte du corps explique donc aisément le fait remarquable que l'une des dernières volontés qui revient le plus fréquemment dans la bouche de ces héros revêt le caractère d'un ultime pacte engageant les survivants à s'assurer que la dépouille ne sera pas abandonnée sans sépulture. Cette inquiétude contractée à l'égard de son moi physique qui n'est plus, outre qu'elle tend à prévenir tout éventuel outrage posthume, ce qui représentait un raffinement d'horreur fort goûté sur la scène tragique, trahit tout d'abord le désir de ne point cesser d'oc- cuper parmi les vivants un certain espace, fût-il aussi infime que celui d'une tombe, laquelle, désignant le moi qui n'est plus, sera prétexte à un nouveau culte, non plus du corps, mais de la tombe. Le moi, mort, n'en continue donc pas moins à vouloir se signifier; et le souci contracté à l'égard du bien-être de ce moi mort qui, d'ailleurs, selon des conceptions fort vieilles, passait pour vivre dans le tombeau, fit que l'on pourvut scrupuleusement, dès la plus haute Antiquité (voir Chadwick, 1959, p. 269278), aux besoins les plus élémentaires du moi physique. Aussi le tombeau renfermaitil un «moi qui vivait» (Sophocle, Électre, v. 1419), et qui, en conséquence, avait gardé ses appétits et passions terrestres; un moi qui avait besoin d'armes, de vases, de vêtements, de parures ${ }^{12}$, et dont on étanchait la soif ou apaisait la faim en répandant du vin, du miel, du lait ou autres mets ${ }^{13}$, mais qui, en même temps, n'hésitait pas à se faire malfaisant ${ }^{14}$ dès que, impie, on venait à négliger les devoirs ou les honneurs qui lui revenaient. Inversement, à partir du moment où l'on se conciliait la «bienveillance» de cet autre moi redoutable (Platon, République, v. 427 b), il devenait un dieu titulaire, prenait part aux affaires de sa propre famille et de celle-ci assurait la protection. Ainsi le je, mort, devenait-il une divinité infernale, qui réclamait ses propres sacrifices d'esclaves et de chevaux (voir Homère, Iliade, XXI, v. 26-28; XXIII, v. 165-176; Virgile, Énéide, X, v. 517-520; XI, v. 197-199); et il n'était point rare d'invoquer le mort en tant que telle, de lui adresser des prières afin qu'il vînt en aide aux malheureux ou qu'il leur fût propice ${ }^{15}$.
La piété collective, d'autre part, entourait de soins tout particuliers les rites mêmes de la sépulture: c'était une «loi générale des Grecs » (nómos panellhnon; voir Euripide, Suppliantes, v. 311, 526, 671; Phéniciennes, v. 1320-1321), et la moindre infraction en la matière exposait les coupables à la vengeance divine (voir Homère, Iliade, XXII, v. 358; Odyssée, XI, v. 72) ou tombait sous le coup de la loi (voir Xénophon, Helléniques, I, 7 ; Démosthène, "Contre Macart», dans Plaidoyers civils, v. 58-59). Car les morts privés de sépulture ou de larmes, et là est l'origine et de la croyance aux revenants (voir Euripide, Troyennes, v. 1085; Hérodote, Histoires, V, v. 92; Virgile, Énéide, VI, v. 371, 379; Cicéron, Tusculanes, I, v. 16) et, sans doute aussi, des pratiques d'incinération telles qu'elles apparaissent chez Homère ${ }^{16}$, étaient d'une certaine manière frappés de proscription: sous forme de larve ou de fantôme, l'âme, malheureuse et irritée contre ceux qui avaient négligé les honneurs funèbres (ktérea), seul garant du repos des trépassés, errait au bord du Styx, devenait malfaisante, hantait les vivants, leur envoyait de terribles maux, ravageait leurs moissons ou frappait de stérilité leur $\mathrm{sol}^{17}$; et ce, jusqu'au jour où l'on entreprenait de lui rendre les derniers honneurs. Voilà pourquoi d'ailleurs les mères des Sept Chefs tombés sous les murs de Thèbes réclamaient les cadavres de leurs fils, abandonnés sans sépulture: [elles voudraient avoir entre les mains] «les corps vigoureux de [leurs] morts, vagabonds sans demeure dernière » (Euripide, Suppliantes, v. 61-62). On comprend ainsi les griefs de l'ombre de Polydoros, qui nous dit sa peine: "Me voici gisant, tantôt sur la grève, tantôt dans les vagues du large, longuement ballotté par le va-et-vient des flots, privé de larmes et privé de sépulture $[. .$.$] ; je voltige, ayant abandonné mon$ corps, et voici deux jours que je flotte dans les airs [...]»(Euripide, Hécube, v. 28 s.). On se souvient également de la recommandation faite par l'ombre de Patrocle au fils de Pélée: "Ensevelis-moi au plus vite, afin que je passe les portes d'Hadès. Des âmes sont là, qui m'écartent, m'éloignent, ombres de défunts. Elles m'interdisent de franchir le fleuve et de les rejoindre, et je suis là, à errer vainement à travers les demeures d'Hadès [...]» (Homère, Iliade, XXIII, v. 71 s.). Et Héliodore fera dire à son Théagène, qui croit entendre gémir l'ombre de sa bien-aimée: "[Très chère âme], je vois bien que tu erres encore sur la terre, ne pouvant te résoudre à quitter un si beau corps d'où la violence t'a arrachée. Peut-être aussi, pour n'avoir pas été inhumée, tu es repoussée par les ombres des enfers ${ }^{18}$. »Cela étant, il ne suffisait 
guère de mettre en terre le cadavre, sans tambour ni trompette; encore fallait-il que les rites et les formules de la cérémonie funèbre fussent observés à la lettre: on se souvient que Plaute met dans la bouche de son revenant des propos qui attestent la peine de celui dont l'âme erre au bord du Styx, pour s'être attachée à un corps qu'un assassin avait mis en terre sans que les rites de la sépulture fussent accomplis (Mostellaria, II, 3).

C'est dire l'importance d'une cérémonie qui, tout à la fois, garantissait la paix aux morts et aux vivants; et il n'était d'ailleurs de pires imprécations que celles où l'on souhaitait à son ennemi d'être privé de sépulture (voir Virgile, Énéide, IV, v. 620). La loi de son côté, jalouse de rigueur, infligeait aux criminels la privation de la sépulture comme le pire des châtiments ${ }^{19}$ et les condamnait à être précipités dans un gouffre (bárathron) ${ }^{20}$ destiné à recevoir leurs dépouilles. Ces convictions expliquent la forte récurrence, dans le discours des

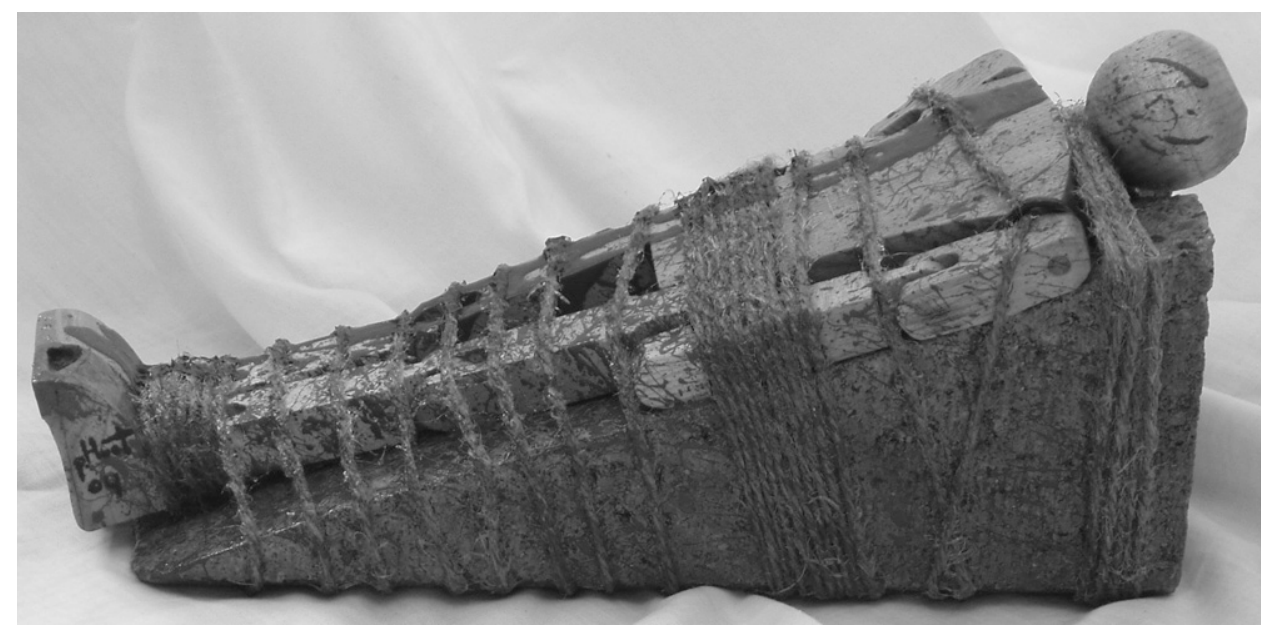

mourants (voir Homère, Iliade, XXII, v. 333-344; Sophocle, Antigone, v. 467; Virgile, Énéide, IX, v. 213), des demandes de sépulture, qui disent la crainte d'être enterré sans que la cérémonie funèbre soit observée ou, pire encore, d'être abandonné en proie aux chiens et aux oiseaux carnassiers. Ajax par exemple, ce «vivant bouclier», comme on l'a souvent décrit, près de se donner la mort, invoque Zeus pour qu'il envoie Teucer, son propre frère, découvrir le premier son cadavre, «avant qu'un de [ses] ennemis, déclare-t-il, ne [l'] ait découvert et ne [le] jette aux chiens et aux oiseaux» (Sophocle, Ajax, v. 828). Le Polynice du même poète, frappé de la malédiction d'Edipe, et sachant que son expédition sous les murs de Thèbes risque d'échouer, exhorte ses sœurs, Antigone et Ismène, à lui assurer les derniers honneurs: "Ô mes sœurs, implore-t-il, vous qui avez entendu ces cruelles malédic- tions paternelles [...], si [les malédictions d'Edipe] s'accomplissent [...], ne me jugez pas indigne d'un tombeau et d'offrandes funéraires » (Edipe à Colone, v. 103-107). Les offrandes étant, bien entendu, la perpétuation des rapports du moi avec autrui. Ailleurs, le même Polynice, mourant cette fois, renouvelle son ultime prière: "Je suis perdu, rapporte un messager, mère, j'ai pitié de toi, de ma sœur [...]. Ensevelissezmoi, ô ma mère » ; et tient à être enveloppé de la terre natale: "Apaisez la ville irritée, pour que j'obtienne au moins un peu de sol natal» (Euripide, Phéniciennes, v. 1437 s.).

Cela dit, les dernières volontés peuvent aussi prolonger les passions, au sens étymologique du terme ( $\mathrm{du}$ grec pathos, affection, sentiment), du moi mourant, et ce, soit directement ou par procuration, comme dans le cas des vœux de vengeance, soit au moyen d'un acte dicté par les impératifs mêmes de l'éthique de telle ou telle passion, tel l'amour notamment.

La soif vengeresse du moi mis à mort, exacerbée dans la mort et prolongeant les passions du moi vivant, réclamait précisément le sang de ses meurtriers et accablait de force menaces et invectives ses contempteurs (voir Sophocle, Électre, v. 1419-1421; Trachiniennes, v. 1201-1202). Mais léguer les soins de sa vengeance, dut-on s'en remettre aux charges de quelque divinité, à quelque généreux justicier, ou encore aux déesses mêmes commises à ces charges, revient aussi à se poser en tant que sujet agissant $a$ posteriori dans le moi d'un autre. Cette procuration, forte du concours de la promesse nietzschéenne, fait du héros survivant le justicier paradigmatique. Ainsi Cassandre, dont on connaît bien les disgrâces, et qui, se voyant au bord de la mort, proteste: «Je ne veux pas chanter mon propre thrène. Au soleil - face à sa clarté suprême j'adresse ma prière: puissent mes vengeurs comme mes meurtriers payer ensemble la dette de l'esclave morte ici, qui fut une proie si facile» (Eschyle, Agamemnon, v. 1322-1326) ${ }^{21}$; et Ajax qui, prêt à se tuer, invoque précisément les Érinyes, déesses de la vengeance, en ces termes: «Et j'invoque encore, pour qu'elles m'assistent, les vierges éternelles [...] Qu'elles sachent comment je succombe, malheureux, sous les fils d'Atrée, et qu'elles les saisissent, eux aussi, à leur tour, pour les faire périr, périr tout entiers, misérables, misérablement [...] » (Sophocle, Ajax, v. 835-839).

Or, il faut inscrire ces vœux et invocations dans leur cadre idéologique propre. Les Anciens étaient-ils indifférents aux imprécations des mourants? Du tout ${ }^{22}$ ! Ces dernières passaient au contraire pour autant d'oracles émanant d'êtres s'apprêtant à devenir, ainsi que nous venons de l'indiquer, autant de génies titulaires investis d'un pouvoir si redoutable. Cette conviction est clairement attestée dans Lysias, qui nous rapporte d'ailleurs que Dionysodoros, au moment de mourir, adressait à son épouse, "qu'il croyait enceinte», des recommandations pour que, s'il lui naissait un fils, elle élevât celui-ci dans la pensée de la vengeance (Discours, t. I, XIII, v. 41-42). Aux XVI et $\mathrm{XVII}^{\mathrm{e}}$ siècles en Europe, les dispositions testamentaires entérineront ces mêmes vieilles conceptions; et, dans son Usage des passions, Senault parlera à son tour des ravages de la haine vengeresse, passion « opiniâtre» et «hardie», et qui, inassouvie, se transmet par voie de succession (Seconde partie, $1^{\text {er }}$ Traité, v. 191-192). La tragédie baroque ne manquera pas de mettre en scène des héros qui, mourants, s'empressent de léguer les soins d'une illustre vengeance, soit à leur généreuse et vaillante descendance, ou à d'autres personnages se disant prêts à travailler à apaiser leurs mânes ${ }^{23}$.

Par ailleurs, l'éthique de l'amour tragique dicte au héros survivant de ne point survivre à son objet d'amour. Formuler le vœu, toujours d'ailleurs sanctionné par le geste, de suivre ce dernier dans la mort n'est donc pas un simple geste de générosité, mais adhésion aux impératifs d'une vieille conviction idéologique qui fait des amants, suivant le mythe de l'espèce androgyne, un tout indivisible: le moi aimant est donc à la fois «moi-même», sur le plan spirituel, et "un autre», sur le plan physique. Or, si la mort peut atteindre ce dernier, elle trouble tout au plus l'harmonie dans le moi spirituel, qui réclame la réunion des amants, et dans la vie et dans la mort, comme véhicule de son expression. Ces considérations expliquent en effet les fréquents suicides d'amour dans l'Antiquité comme dans la tragédie française: Didon, Cleité, Pauline, Porcie, Cléopâtre, Sophonisbe, Massinisse, 
Panthée, pour ne citer que les personnages les plus célèbres ${ }^{24}$, sont au nombre des héros dont le «beau désespoir» illustre ces convictions idéologiques. Les héros de la scène française du XVII ${ }^{\mathrm{e}}$ siècle seront tout aussi animés par les mêmes soins : ainsi Bradamante et Roger, «beau couple» réuni dans "un même tombeau ", et sous une "même lame funèbre» (Anonyme, La mort de Bradamante, fin); Camille et Cuirace, dont les corps vont également être enfermés "en un même tombeau» (Corneille, Horace, fin), et enfin, Porcie et Brute, ces "généreux amants» qui, conformément aux ordres du magnanime Antoine, recevront sur un même bûcher «les derniers de leurs embrassements» (Guérin de Bouscal, La mort de Brute et de Porcie, fin). Cette réunion, deuxième guérison de l'espèce androgyne d'après le discours d'Aristophane dans le Banquet de Platon, n'étant plus sujette à l'incertitude de l'avenir, consacre le triomphe définitif du moi aimant: l'au-delà en définit l'espace, et l'éternité, la durée.

En somme, les dernières volontés furent l'occasion, pour les héros de la tragédie grecque, de dire les agitations du moi tragique in articulo mortis. Moyennant l'accomplissement d'un geste sacramentel qui tient lieu d'une promesse se dressant contre les incertitudes du devenir, elles permirent en effet aux héros tragiques de dire le culte du moi physique, de prévenir l'attentat à l'intégrité de ce dernier, d'en prolonger les passions soit directement ou par procuration, comme dans le cas des vœux de vengeance, où le mourant devient $a$ posteriori sujet agissant dans le moi d'un autre sujet, soit au moyen d'un acte dicté par les impératifs mêmes de l'éthique de telle ou telle passion, telle que l'amour, qui, suivant sa définition mytho-idéologique, fait du sujet aimant à la fois un moi-même et un autre.

\section{Bibliographie}

ANONYME (1624). "La mort de Bradamante», Théâtre Français, Paris, Paul Manian.

ANONYME (1624). "La mort de Roger», Théâtre Français, Paris, Paul Manian.

APOLLONIOS DE RHODES (1974). Argonautiques, texte établi et commenté par F. VIAN, trad. E. DELGE, Paris, Les Belles Lettres.

ARISTOPHANE (1962-1964). CEuvres, texte établi par V. COULON, trad. H. VAN DAELE, Paris, Les Belles Lettres, 5 vol.

BENSERADE, Isaac de (1636). La mort d'Achille et la dispute de ses armes, Paris, Antoine de Sommaville.

BERGERAC, Cyrano de (1982 [1653]). «La mort d'Agrippine», dans C.J. GOSSIP (dir.), Textes littéraires, $\mathrm{n}^{\circ} 44$, Exeter University Printing Unit.
BORÉE, V. (1627). Les princes victorieux, tragédies françoises (Achille; Amé le Grand; Béral; Rhode; Tomyre), Lyon, V. de Coeursilly.

BOUSCAL, Guérin de (1637). La mort de Brute et de Porcie ou la vengeance de la mort de César, Paris, Toussainct Quinet.

CHADWICK, J. (1959). «Minoan Linear A», Antiquity, no 33, p. 269-278.

CICÉRON (1970). Tusculanes, G. FOHLEN (dir.), trad. J. HUMBERT, Paris, Les Belles Lettres, $2 \mathrm{t}$.

CORNEILLE, Pierre (1963). EEures complètes, A. STEGMANN (dir.), préf. R. LEBÈGUE, Paris, Seuil.

DARESTE, R., B. HAUSSOULLIER et T. REINACH (dir.) (1965). Recueil des inscriptions juridiques grecques, 2 vol. en 24 , Rome.

DÉMOSTHÈNE (1950-1960). Plaidoyers civils, texte établi et trad. par L. GERNET, Paris, Les Belles Lettres, 4 t.

DIEPOLDER, Hans (1931). Die attischen Grabreliefs des 5. und 4. Jahrhunderts v. Chr., Berlin.

ÉPICTÈTE (1962). Entretiens, texte établi et trad. par J. SOUILHÉ, Paris, Les Belles Lettres, $4 \mathrm{t}$.

ESCHYLE (1969). Tragédies, texte établi et trad. par P. MAZON, Paris, Les Belles Lettres, 2 t.

EURIPIDE (1959-1961). Tragédies, Paris, Les Belles Lettres, 7 t. : t. I (1961) et II (1960), texte établi et trad. par L. MÉRIDIER, t. III et IV (1959), par L. PARMENTIER et H. GRÉGOIRE, t. V (1961) par H. GRÉGOIRE et L. MÉRIDIER, t. VI (1961) par H. GRÉGOIRE avec le concours de J. MEUNIER, t. VII (1959) par F. CHAPOUTHIER et L. MÉRIDIER.

GRENAILLE, François (1639). L'innocent malheureux ou La mort de Crispe, Paris, Jean Pâle.

HARDY, Alexandre (1884). Le théâtre d'Alexandre Hardy, Erster Neudruck der Dramen von Pierre Corneille's unmittelbarem Vorläufer nach den Exemplaren der Dresdener, Münchener und der Wolfenbütteler Bibliothek, von E. Stengel, Marburg, 5 t.

HÉLIODORE D'ÉMÈSE (1991). Les Éthiopiques (Théagène et Chariclée), texte établi par R.M. RATTENBURY et Rev. T.W. LUMB, trad. J. MAILLOIN, Paris, Les Belles Lettres.

HÉRODOTE (1964). Histoires, texte établi et trad. par P.-E. LEGRAND, Paris, Les Belles Lettres, 11 vol.

HÉSIODE (1972). Théogonie, texte établi et trad. par P. MAZON, Paris, Les Belles Lettres.

HOMÈRE (1987). Iliade, texte établi et trad. par P. MAZON, Paris, Les Belles Lettres, 4 t.

HOMÈRE (1963). Odyssée, texte établi et trad. par V. BÉRARD, Paris, Les Belles Lettres, $3 \mathrm{t}$.
ISOCRATE (1956-1962). Discours, texte établi et trad. par G. MATHIEU et E. BREMOND, Paris, Les Belles Lettres, 4 vol.

LA CALPRENÈDE, Gautier de Costes, sieur de (1986 [1636]). «La mort de Mithridate», dans J. SCHERER (dir.), Théâtre du XVII ${ }^{e}$ s., Paris, Gallimard.

LA ROCHEFOUCAULD, François de (1977). Maximes, J. TRUCHET (dir.), Paris, Garnier.

LUCAIN (1947). La Guerre civile (La Pharsale), A. BOURGERY (dir.), Paris, Les Belles Lettres, $2 \mathrm{t}$

LYSIAS (1967). Discours, texte établi et trad. par L. GERNET et M. BIZOS, Paris, Les Belles Lettres, $2 \mathrm{t}$.

MOURLON BEERNAERT, P. (1963). «Les premiers lyriques grecs en face de la mort », Études classiques, vol. 31, no 4 .

OVIDE (1990). Fastes, trad. et annoté par H. LE BONNIEC, Paris, Les Belles Lettres.

PERSE (1995). Satires, présentation, trad. et notes de B. PAUTRAT, Paris, La Salamandre.

PLATON (1951-). Euvres complètes, Paris, Les Belles Lettres.

PLAUTE (1970). Mostellaria, texte établi et trad. par A. ERNOUT, Paris, Les Belles Lettres.

PLINE LE JEUNE (1953-1959). Lettres, texte établi et trad. par A.-M. GUILLEMIN, Paris, Les Belles Lettres, 4 vol.

PLOTIN (1953-1963). Ennéades, texte établi et trad. par É. BRÉHIER, Paris, Les Belles Lettres, 6 vol. en 7.

PLUTARQUE (1971). Les œuvres morales et mêlées de Plutarque, trad. J. AMYOT, Mouton Éditeur.

PLUTARQUE (1957). Vies, Paris, Les Belles Lettres, coll. «G. Budée ».

ROHDE, E. (1928). Psyché. Le culte de l'âme chez les Grecs et leur croyance à l'immortalité, éd. fr. par A. REYMOND, Paris, Payot.

ROTROU, Jean (1820). CEuvres de J. Rotrou, Paris, Th. Desoer.

SCHLIEMANN, H. (1976 [1880]). Mycenae: A Narrative of Researches and Discoveries at Mycenae and Tiryns, New York, Arno Press.

SENAULT, J.-F. (1987 [1650]). De l'usage des passions, Paris, Fayard.

SÉNÈQUE (1964). Tragédies, L. HERMANN (dir.), Paris, Les Belles Lettres, 2 t.

SÉNÈQUE (1951). Dialogues, A. BOURGERY (dir.), Paris, Les Belles Lettres, 4 t.

SOPHOCLE (1964). Tragédies, texte établi et trad. par P. MAZON, Paris, Les Belles Lettres, 3 t.

THÉOGNIS DE MÉGARE (1975). Poèmes élégiaques, texte établi et trad. par J. CARRIÈRE, Paris, Les Belles Lettres.

THUCYDIDE (1958-). La Guerre du Péloponnèse, texte établi et trad. par J. de ROMILLY, Paris, Les Belles Lettres.

VAN GENNEP, A. (1981 [1909]). Les rites de passage, Paris, Picard. 
VIRGILE (1964). Énéide, H. GOELZER et A. BELLESORT (dir.), Paris, Les Belles Lettres, $2 \mathrm{t}$.

XÉNOPHON (1971-1973). Cyropédie, texte, trad. et commentaire de M. BIZOS et G. ROCHEFORT, t. I et II, Paris.

XÉNOPHON (1965-1966 [1936-1954]). Helléniques, texte, trad. et commentaires J. HATZFELD, Paris, 2 vol.

\section{Notes}

1. On verra s'accomplir le même geste sur la scène française. Ainsi, chez Hardy, dans $L a$ mort d'Achille (IV, 2), dans La mort de Daire $(\mathrm{V}, 1)$ et dans Alcméon (IV).

2. Voir aussi le cas de la Médée d'Apollonios de Rhodes, laquelle, au bord du suicide, se rappelle, elle aussi, les joies les plus humbles de la vie: «[...] la vue du soleil lui parut plus douce qu'autrefois [...]» (Argonautiques, chant III, v. 815).

3. Chez Sénèque, le même Héraclès adresse ses toutes premières paroles, dès son retour à la face des cieux, à Phébus (Hercule furieux, v. 592). Quant à Thésée, qui avait accompagné le fils d'Alcmène dans sa descente aux Enfers, il nous dit, dans une autre pièce du même poète, sa joie de revoir la lumière, laquelle, pourtant, lui "émousse la vue » (Phèdre, v. 835-837). Ailleurs, le même Thésée insiste sur les méfaits des ténèbres sur sa vue (voir Hercule furieux, v. 652-653).

4. Pour d'autres témoignages, recueillis chez les premiers lyriques grecs, voir P. Mourlon Beernaert, 1963, p. 363 ; Théognis, Poèmes élégiaques, I, v. 569.

5. Lidée se trouve déjà dans Hésiode (voir Théogonie, v. 758 s.).

6. L'horrible aspect de Cerbère, gardien des Enfers, constitue à lui seul une souillure pour le regard (voir Sénèque, Hercule furieux, v. 593-605).

7. Même souci pour Hélène, laquelle, envisageant le suicide, cherche le meilleur moyen de se donner une mort qui ne porte pas préjudice à son éclatante beauté: "Comment donc mourir en beauté ? Grotesque, la pendaison qui vous suspend au bout d'une corde chez les esclaves est tenue pour avilissante; le poignard a quelque chose de fier et d'élégant [...]» (Euripide, Hélène, v. 300 s.).

8. Sénèque conservera à la vierge troyenne cette même pudeur, tout en soulignant davantage sa beauté. Voir Troades, v. 1137-1139.

9. On sait que le fils d'Alcmène était devenu, par la gloire de ses douze travaux accomplis, le modèle même de la sagesse, tant pour les cyniques que pour les stoïciens (voir par exemple, chez Épictète, Entretiens. t. I, 6, 32-36; t. II, 16, 44; t. III, 22, 57; 24,13; 26, 31-32).

10. Voir Hercule sur l'Eta, v. 1740 s. Il convient de songer ici au récit des immolations volontaires et héroïques des soldats de Lucain, lesquels, vaincus, et désirant ravir à l'ennemi le plaisir de les tuer, vont allégrement, et à la manière de l'Hercule latinisé, au-devant de la mort. Voir La Guerre civile (La Pharsale), t. I, Liv. II, 154-159.
11. Cette définition de la belle mort rejoint celle qui, plus tard, sera nommée par les chrétiens «la mort douce», dont seuls les bienheureux pourront jouir. Voilà pourquoi d'ailleurs lorsqu'il s'agit de décrire la mort d'un impie, tel l'Égisthe d'Euripide par exemple, c'est sous le signe de fureur, de spasmes ou d'accès de convulsions que l'on nous rapporte sa déshonorante et scandaleuse chute: le traître et efféminé Égisthe, agonisant, «tout son corps, dit un messager, de haut en bas, s'agite en convulsions. Il pousse un grand cri et se tord dans les affres de la mort » (Électre, v. 840 s.). Parallèlement, la prodigieuse mort du malheureux Edipe, devenu enfin bienheureux, nous est rapportée en des termes qui font songer aux «belles morts des âmes vertueuses»: «Il n'est pas parti escorté de plaintes, ni dans les souffrances de la maladie, mais en plein miracle, s'il en fut jamais de tel pour un homme » (Sophocle, Edipe à Colone, v. 1663-1665).

12. Tous ces objets, et bien d'autres, on les disposait autour de la dépouille à l'intérieur de la tombe. Voir à ce sujet Schliemann, 1976.

13. On faisait parvenir ces oblationes pro defunctis à la faveur d'un fossé creusé près du tombeau. Voir Euripide, Iphigénie en Tauride, v. 163; v. 633 s.; Virgile, Énéide, V, v. 76-80, VI, v. 225. Il faut se garder toutefois de confondre cet usage avec celui des repas funèbres (táfon dainúnai, voir Homère, Iliade, XXIII, v. 29; XXIV, v. 802), où, à certains jours de l'année, après avoir prononcé certaines formules consacrées, l'on conviait les morts. Voir Eschyle, Choéphores, v. 162; v. 482-484; Euripide, Iphigénie en Tauride, v. 157-163; Hécube, v. 563 ; Électre, v. 505 s. ; Perse, Satires, v. 619-620. Voir aussi Ovide (Fastes, V, v. 429 s.), qui, évoquant la puissance des morts, nous parle des gestes apotropiques et des précautions qu'il fallait prendre au moment où chaque père de famille devait, le 9 , le 11 et le 13 mai de chaque année, faire des offrandes pour apaiser les lémures, qui sont les morts malfaisants.

14. Eschyle, Choéphores, v. 22 s.; v. 322-327; Sophocle, Ajax, v. 1154-1155; Xénophon, Cyropédie, VIII, 7, 18; Hérodote, Histoires, I, 167; Plaute, Mostellaria, II, 2, 68; Ovide, Fastes, II, 549-556; Pline le J., VII, 27 ; Plotin, Ennéades, IV, 7, 15. Le repos des défunts était si étroitement associé à ces devoirs funèbres que diverses dispositions testamentaires de l'époque durent s'en ressentir. Voir par exemple les fondations testamentaires (t. II, $n^{\circ}$ XXIV) dans Dareste, Haussoullier et Reinach, 1965. Voir sur ce sujet, Rohde, 1928, p. 172.

15. Voir Eschyle, Choéphores, v. 130-148; v. 479 s.; Sophocle, Électre, v. 453-458; Euripide, Alceste, v. 1004-1016; Thucydide, La Guerre du Péloponnèse, III, v. 59.

16. L'incinération, rite essentiel de passage, marquait en effet la séparation définitive de l'âme d'avec le corps, condition essentielle au repos des trépassés. Voir Homère, Iliade, VII, v. 410 ; VIII, v. 79 s.; XXII, v. 338 s.; XXXIII, v. 76 ; Odyssée, XI; v. 51 s.; v. 218-222. Sur cette question, voir Rohde, 1928, p. 19 et s. ; voir aussi, pour les rites de passage en général, Van Gennep, 1981, p. 209-236.
17. Le refus de la sépulture passait en effet pour être la source de pires épreuves: «[...] ce n'est pas un mal égal ni semblable que les morts soient privés de sépulture et que les vivants soient dépouillés de leur patrie; l'un est plus grave pour ceux qui empêchent la sépulture que pour ceux qui subissent ce malheur", Isocrate, Discours, XIV, v. 55.

18.Éthiopiques, II, V, 2. Voir aussi Liv. VI, t. II, $\mathrm{XV}, 2$; Homère, Odyssée, XI, 73 s.; Lysias, Discours, II, v. 7 ; Euripide, Troyennes, v. 1085 ; Virgile, Énéide, VI, v. 331; v. 371; Cicéron, Tusculanes, I, v. 44; Pline le J., Lettres, VII, 27, 11.

19. Voir Eschyle, Sept contre Thèbes, v. 1013 ; Sophocle, Antigone, v. 189; Euripide, Phéniciennes, v. 1627 ; Lysias, "Oraison funèbre ", in Discours, v. 7-9; Platon, Lois, v. 909 c.

20. Voir Thucydide, La Guerre du Péloponnèse, II, 67, 4; Xénophon, Helléniques, I, 20 ; Plutarque, "Thémistocle», in Vies, 22.

21. «Texte conjectural», note le traducteur. L'idée exprimée par Cassandre est sans doute qu'elle souhaite voir ses ennemis "payer ensemble » à ses vengeurs «le meurtre de l'esclave qu'elle est devenue».

22. La tragédie baroque en France emboîtera le pas. Au moment de sa mort, Patrocle prophétise la défaite de son vainqueur: "Je te veux annoncer ta prochaine défaite,/ Sache donc, cruel, qu'Achille, en peu de temps,/ Te doit priver du lot qu'au Monde tu prétends,/ Qu'il te doit, me vengeant, faire passer la rive,/ Et les sombres marrées, d'où personne n'arrive » (Borée, Achille, dans Les princes victorieux, II, 2). Achille lui-même $(\mathrm{V}, 2)$ fera à son tour l'objet d'une semblable prophétie placée dans la bouche de sa victime, Mémnon, au moment où elle expire: «Or, premier que passer l'Achéronide bord,/ En peu de mots je vais t'avertir de ton sort,/ Sache donc, cruel, que jamais Polyxène,/ N'allégera les fers de l'amour qui te gêne,/ Et que bientôt Pâris te fera trébucher $[\ldots]$ » On connaît la suite. Ailleurs, les détails d'une bataille aussi célèbre que celle de Pharsale sont prévus dans les imprécations adressées par Mithridate à l'encontre du traître Pharnace (La Calprenède, La mort de Mithridate, IV, 3).

23. Voir Hardy, La mort de Daire, V, 2; La mort d'Achille, IV, 2; Benserade, La mort d'Achille, IV, 6; Rotrou, Hercule mourant, IV, 4; Grenaille, L'innocent malheureux, V, 8; Anonyme, La mort de Roger, II, 3 ; Corneille, Médée, V, 5 ; La mort de Pompée, II, 2; Rodogune, V, 4; Cyrano de Bergerac, La mort d'Agrippine, IV, 1.

24. Notons aussi le cas, un peu moins connu, mais non pas moins digne d'intérêt, d'Évandé, veuve de Capanée, laquelle se précipite dans le bûcher de celui-ci afin de n'en être point séparée par la mort: "C'est ici que m'attend un illustre triomphe [...] Je meurs auprès de mon époux. [...] Je m'élance dans le bûcher de Capanée. [...] Ô bonheur [...], pour moi, pour mon époux, auquel va m'unir ce brasier!» Une indication scénique précise: «Elle se précipite» (Euripide, Suppliantes, v. 10591071 passim). 